Draft version OCtober 30, 2018

Preprint typeset using IATEX style emulateapj v. 08/13/06

\title{
THE IMPORTANCE OF MAGNETIC-FIELD-ORIENTED THERMAL CONDUCTION IN THE INTERACTION OF SNR SHOCKS WITH INTERSTELLAR CLOUDS
}

\author{
S. Orlando ${ }^{1}$, F. Bocchino ${ }^{1}$ \\ INAF - Osservatorio Astronomico di Palermo "G.S. Vaiana", Piazza del Parlamento 1, 90134 Palermo, Italy \\ F. ReAle ${ }^{2,1}$, G. Peres ${ }^{2,1}$ And P. Pagano \\ Dip. di Scienze Fisiche \& Astronomiche, Univ. di Palermo, Piazza del Parlamento 1, 90134 Palermo, Italy \\ Draft version October 30, 2018
}

\begin{abstract}
We explore the importance of magnetic-field-oriented thermal conduction in the interaction of supernova remnant (SNR) shocks with radiative gas clouds and in determining the mass and energy exchange between the clouds and the hot surrounding medium. We perform 2.5D MHD simulations of a shock impacting on an isolated gas cloud, including anisotropic thermal conduction and radiative cooling; we consider the representative case of a Mach 50 shock impacting on a cloud ten-fold denser than the ambient medium. We consider different configurations of the ambient magnetic field and compare MHD models with or without the thermal conduction. The efficiency of the thermal conduction in the presence of magnetic field is, in general, reduced with respect to the unmagnetized case. The reduction factor strongly depends on the initial magnetic field orientation, and it is minimum when the magnetic field is initially aligned with the direction of shock propagation. The thermal conduction contributes to suppress hydrodynamic instabilities, reducing the mass mixing of the cloud and preserving the cloud from complete fragmentation. Depending on the magnetic field orientation, the heat conduction may determine a significant energy exchange between the cloud and the hot surrounding medium which, while remaining always at levels less than those in the unmagnetized case, leads to a progressive heating and evaporation of the cloud. This additional heating may contrast the radiative cooling of some parts of the cloud, preventing the onset of thermal instabilities.

Subject headings: conduction — magnetohydrodynamics — shock waves — ISM: clouds - ISM: magnetic fields - ISM: supernova remnants
\end{abstract}

\section{INTRODUCTION}

The interaction of the shock waves of supernova remnants (SNRs) with the magnetized and inhomogeneous interstellar medium (ISM) is responsible of the great morphological complexity of SNRs and certainly plays a major role in determining the exchange of mass, momentum, and energy between diffuse hot plasma and dense clouds or clumps. These exchanges may occur through, for example, hydrodynamic ablation and thermal conduction and, among other things, lead to the cloud crushing and to the reduction of the Jeans mass causing star formation.

The propagation of hot SNR shock fronts in the ISM and their interaction with local over-dense gas clouds have been investigated with detailed hydrodynamic and MHD modeling. The most complete review of this problem in the unmagnetized, non-conducting, and nonradiative limits is provided by Klein et al. (1994). These studies have shown that the cloud is disrupted by the action of both Kelvin-Helmholtz (KH) and Rayleigh-Taylor (RT) instabilities after several crushing times, with the cloud material expanding and diffusing into the ambient medium. An ambient magnetic field can both act as a confinement mechanism of the plasma and be modified by the interstellar flow and by local field stretching. Also, a strong magnetic field is known to limit hydrodynamic instabilities developing during the shock-cloud interac-

\footnotetext{
${ }^{1}$ Consorzio COMETA, via Santa Sofia 64, 95123 Catania, Italy

2 INAF, Viale del Parco Mellini 84, 00136 Roma, Italy
}

tion by providing an additional tension at the interface between the cloud and the surrounding medium (e.g Mac Low et al. 1994; Jones et al. 1996).

The interaction of the shock with a radiative cloud has been only recently analyzed in detail (e.g. Mellema et al. 2002; Fragile et al. 2004). 2D calculations have shown that the effect of the radiative cooling is to break up the clouds into numerous dense and cold fragments that survive for many dynamical timescales. In the case of the interaction between magnetized shocks and radiative clouds, the magnetic field may enhance the efficiency of the radiative cooling, influencing the size and distribution of condensed cooled fragments (Fragile et al. 2005).

The role played by the thermal conduction during the shock-cloud interaction has been less studied so far. In a previous paper, Orlando et al. (2005) (hereafter Paper I) have addressed this point in the unmagnetized limit. In particular, we have investigated the effect of thermal conduction and radiative cooling on the cloud evolution and on the mass and energy exchange between the cloud and the surrounding medium; we have selected and explored two different physical regimes chosen so that either one of the processes is dominant. In the case dominated by the radiative losses, we have found that the shocked cloud fragments into cold, dense, and compact filaments surrounded by a hot corona which is ablated by the thermal conduction. Instead, in the case dominated by thermal conduction, the shocked cloud evaporates in a few dynamical timescales. In both cases, we have found that 
the thermal conduction is very effective in suppressing the hydrodynamic instabilities that would develop at the cloud boundaries, preserving the cloud from complete destruction. Orlando et al. (2006) and Miceli et al. (2006) have studied the observable effects of thermal conduction on the evolution of the shocked cloud in the X-ray band.

Here, we extend the previous studies by investigating the effect of the thermal conduction in a magnetized medium, unexplored so far. Of special interest to us is to investigate the role of anisotropic thermal conduction - funneled by locally organized magnetic fields in the mass and energy exchange between ISM phases. In particular, we aim at addressing the following questions: How and under which physical conditions does the magnetic-field-oriented thermal conduction influence the evolution of the shocked cloud? How do the mass mixing of the cloud material and the energy exchange between the cloud and the surrounding medium depend on the orientation and strength of the magnetic field and on the efficiency of the thermal conduction?

To answer these questions, we take as representative the model case of a shock with Mach number $\mathcal{M}=50$ (corresponding to a post-shock temperature $T \approx 4.7 \times$ $10^{6} \mathrm{~K}$ for an unperturbed medium with $T=10^{4} \mathrm{~K}$ ) impacting on an isolated cloud ten-fold denser than the ambient medium. Paper I has shown that, in this case, the thermal conduction dominates the evolution of the shocked cloud in the absence of magnetic field. Around this basic configuration, we perform a set of MHD simulations, with different interstellar magnetic field orientations, and compare models calculated with thermal conduction turned either "on" or "off" in order to identify its effects on the cloud evolution.

The paper is organized as follows: in Sect. 2 we describe the MHD model and the numerical setup; in Sect. 3 we discuss the results; and finally in Sect. 4 we draw our conclusions.

\section{THE MODEL}

We model the impact of a planar supernova shock front onto an isolated gas cloud. The shock propagates through a magnetized ambient medium and the cloud is assumed to be small compared to the curvature radius of the shock ${ }^{3}$. The fluid is assumed to be fully ionized with a ratio of specific heats $\gamma=5 / 3$. The model includes radiative cooling, thermal conduction (including the effects of heat flux saturation) and resistivity effects. The shock-cloud interaction is modeled by solving numerically the time-dependent non-ideal MHD equations (written in non-dimensional conservative form):

$$
\begin{gathered}
\frac{\partial \rho}{\partial t}+\nabla \cdot(\rho \mathbf{u})=0 \\
\frac{\partial \rho \mathbf{u}}{\partial t}+\nabla \cdot(\rho \mathbf{u u}-\mathbf{B B})+\nabla P_{*}=0 \\
\frac{\partial \rho E}{\partial t}+\nabla \cdot\left[\mathbf{u}\left(\rho E+P_{*}\right)-\mathbf{B}(\mathbf{u} \cdot \mathbf{B})\right]= \\
\nabla \cdot[\mathbf{B} \times(\eta \nabla \times \mathbf{B})]-\nabla \cdot \mathbf{F}_{\mathrm{c}}-n_{\mathrm{e}} n_{\mathrm{H}} \Lambda(T)(3)
\end{gathered}
$$

${ }^{3}$ In the case of a small cloud, the SNR does not evolve significantly during the shock-cloud interaction, and the assumption of a planar shock is justified (see also Klein et al. 1994).

$$
\frac{\partial \mathbf{B}}{\partial t}+\nabla \cdot(\mathbf{u B}-\mathbf{B u})=-\nabla \times(\eta \nabla \times \mathbf{B}),
$$

where

$$
P_{*}=P+\frac{B^{2}}{2}, \quad E=\epsilon+\frac{1}{2} u^{2}+\frac{1}{2} \frac{B^{2}}{\rho},
$$

are the total pressure, and the total gas energy (internal energy, $\epsilon$, kinetic energy, and magnetic energy) respectively, $t$ is the time, $\rho=\mu m_{H} n_{\mathrm{H}}$ is the mass density, $\mu=1.26$ is the mean atomic mass (assuming cosmic abundances), $m_{H}$ is the mass of the hydrogen atom, $n_{\mathrm{H}}$ is the hydrogen number density, $\mathbf{u}$ is the gas velocity, $T$ is the temperature, $\mathbf{B}$ is the magnetic field, $\eta$ is the resistivity according to Spitzer (1962), $\mathbf{F}_{\mathrm{c}}$ is the conductive flux, and $\Lambda(T)$ represents the radiative losses per unit emission measure (e.g. Raymond \& Smith 1977; Mewe et al. 1985; Kaastra \& Mewe 2000). We use the ideal gas law, $P=(\gamma-1) \rho \epsilon$.

In order to track the original cloud material, we use a tracer that is passively advected in the same manner as the density. We define $C_{\mathrm{cl}}$ the mass fraction of the cloud inside the computational cell. The cloud material is initialized with $C_{\mathrm{cl}}=1$, while $C_{\mathrm{cl}}=0$ in the ambient medium ${ }^{4}$. During the shock-cloud evolution, the cloud and the ambient medium mix together, leading to regions with $0<C_{\mathrm{cl}}<1$. At any time $t$ the density of cloud material in a fluid cell is given by $\rho_{\mathrm{cl}}=\rho C_{\mathrm{cl}}$.

The thermal conductivity in an organized magnetic field is known to be highly anisotropic and it can be extraordinarily reduced in the direction transverse to the field. The thermal flux, therefore, is locally split into two components, along and across the magnetic field lines, $\mathbf{F}_{\mathrm{c}}=F_{\|} \mathbf{i}+F_{\perp} \mathbf{j}$, where

$$
\begin{aligned}
& F_{\|}=\left(\frac{1}{\left[q_{\mathrm{spi}}\right]_{\|}}+\frac{1}{\left[q_{\mathrm{sat}}\right]_{\|}}\right)^{-1}, \\
& F_{\perp}=\left(\frac{1}{\left[q_{\mathrm{spi}}\right]_{\perp}}+\frac{1}{\left[q_{\mathrm{sat}}\right]_{\perp}}\right)^{-1},
\end{aligned}
$$

to allow for a smooth transition between the classical and saturated conduction regime. In Eqs. 5, $\left[q_{\text {spi }}\right]_{\|}$and $\left[q_{\text {spi }}\right]_{\perp}$ represent the classical conductive flux along and across the magnetic field lines (Spitzer 1962)

$$
\begin{aligned}
& {\left[q_{\mathrm{spi}}\right]_{\|}=-\kappa_{\|}[\nabla T]_{\|} \approx-5.6 \times 10^{-7} T^{5 / 2}[\nabla T]_{\|}} \\
& {\left[q_{\mathrm{spi}}\right]_{\perp}=-\kappa_{\perp}[\nabla T]_{\perp} \approx-3.3 \times 10^{-16} \frac{n_{\mathrm{H}}^{2}}{T^{1 / 2} B^{2}}[\nabla T]_{\perp}}
\end{aligned}
$$

where $[\nabla T]_{\|}$and $[\nabla T]_{\perp}$ are the thermal gradients along and across the magnetic field, and $\kappa_{\|}$and $\kappa_{\perp}$ (in units of erg s $\mathrm{s}^{-1} \mathrm{~K}^{-1} \mathrm{~cm}^{-1}$ ) are the thermal conduction coefficients along and across the magnetic field lines ${ }^{5}$, respectively. The saturated flux along and across the magnetic field lines, $\left[q_{\text {sat }}\right]_{\|}$and $\left[q_{\text {sat }}\right]_{\perp}$, are (Cowie \& McKee 1977)

\footnotetext{
${ }^{4}$ We checked that the used numerical scheme ensures that always $0 \leq C_{\mathrm{cl}} \leq 1$

${ }_{5}^{5}$ For the values of $T, n_{\mathrm{H}}$ and $B$ used here, $\kappa_{\|} / \kappa_{\perp} \approx 10^{16}$ at the beginning of the shock-cloud interaction.
} 
TABLE 1

SUMMARY OF THE INITIAL PHYSICAL PARAMETERS CHARACTERIZING THE MHD SIMULATIONS.

\begin{tabular}{llcl}
\hline \hline & Temperature & Density & Velocity \\
\hline ISM & $10^{4} \mathrm{~K}$ & $0.1 \mathrm{~cm}^{-3}$ & 0.0 \\
Cloud & $10^{3} \mathrm{~K}$ & $1.0 \mathrm{~cm}^{-3}$ & 0.0 \\
Post-shock medium: & $4.7 \times 10^{6} \mathrm{~K}$ & $0.4 \mathrm{~cm}^{-3}$ & $430 \mathrm{~km} \mathrm{~s}^{-1}$
\end{tabular}

$$
\begin{aligned}
& {\left[q_{\mathrm{sat}}\right]_{\|}=-\operatorname{sign}\left([\nabla T]_{\|}\right) 5 \phi \rho c_{\mathrm{s}}^{3},} \\
& {\left[q_{\mathrm{sat}}\right]_{\perp}=-\operatorname{sign}\left([\nabla T]_{\perp}\right) 5 \phi \rho c_{\mathrm{s}}^{3},}
\end{aligned}
$$

where $c_{\mathrm{s}}$ is the isothermal sound speed, and $\phi$ is a number of the order of unity; we set $\phi=0.3$ according to the values suggested for a fully ionized cosmic gas: $0.24<\phi<0.35$ (Giuliani 1984; Borkowski et al. 1989; Fadeyev et al. 2002, and references therein). As discussed in Paper I, this choice implies that no thermal precursor develops during the shock propagation, consistent with the fact that no precursor is observed in young and middle aged SNRs.

The initial unperturbed ambient medium is magnetized, isothermal (with temperature $T_{\text {ism }}=10^{4} \mathrm{~K}$, corresponding to an isothermal sound speed $c_{\text {ism }}=11.5$ $\mathrm{km} \mathrm{s}^{-1}$ ), and uniform (with hydrogen number density $n_{\text {ism }}=0.1 \mathrm{~cm}^{-3}$; see Table 1 ). The gas cloud is in pressure equilibrium with its surrounding and has a circular cross-section with radius $r_{\mathrm{cl}}=1 \mathrm{pc}$; its radial density distribution is given by

$$
n_{\mathrm{cl}}(r)=n_{\mathrm{ism}}+\frac{n_{\mathrm{cl} 0}-n_{\mathrm{ism}}}{\cosh \left[\sigma\left(r / r_{\mathrm{cl}}\right)^{\sigma}\right]},
$$

where $n_{\mathrm{cl} 0}$ is the hydrogen number density at the cloud center, $r$ is the radial distance from the cloud center and $\sigma=10$. The above distribution describes a thin transition layer $\left(\sim 0.3 r_{\mathrm{cl}}\right)$ around the cloud that smoothly brings the cloud density to the value of the surrounding medium ${ }^{6}$. The initial density contrast between the cloud center and the ambient medium is $\chi=n_{\mathrm{clo}} / n_{\text {ism }}=10$. The cloud temperature is determined by the pressure balance across the cloud boundary.

The SNR shock front propagates with a velocity $w=$ $\mathcal{M} c_{\text {ism }}$ in the ambient medium, where $\mathcal{M}$ is the shock Mach number, and $c_{\text {ism }}$ is the sound speed in the interstellar medium; we consider a shock propagating with $\mathcal{M}=50$, i.e. a shock velocity $w \approx 570 \mathrm{~km} \mathrm{~s}^{-1}$ and a temperature $T_{p s h} \approx 4.7 \times 10^{6} \mathrm{~K}$. As discussed in Paper $\mathrm{I}$, in this case (for a cloud with $r_{\mathrm{cl}}=1 \mathrm{pc}$ and $\chi=10$ ) the cloud dynamics would be dominated by thermal conduction in the absence of magnetic field. The post-shock conditions of the ambient medium well before the impact onto the cloud are given by the strong shock limit (Zel'dovich \& Raizer 1966).

Starting from this basic configuration, we consider a set of simulations with different initial magnetic field orientations. We adopt a 2.5D Cartesian coordinate system $(x, y)$, implying that the simulated clouds are cylinders

\footnotetext{
${ }^{6}$ A finite transition layer, in general, is expected in real interstellar clouds due, for instance, to thermal conduction (Balbus 1986; see also Nakamura et al. 2006).
}

TABLE 2

Summary of the MHD Simulations. In All RUNS THE SHOCK MaCh number is $\mathcal{M}=50$, the Density CONTRAST is $\chi=10$, AND THE CLOUd CRUSHING TIME IS $\tau_{\mathrm{cc}} \approx 5.4 \times 10^{3} \mathrm{YR}$

\begin{tabular}{lcccccc}
\hline \hline \multicolumn{1}{c}{ Run } & $\begin{array}{c}|\mathbf{B}| \\
\mu \mathrm{G}\end{array}$ & $\beta_{0}$ & $\begin{array}{c}\text { Field } \\
\text { Comp. }\end{array}$ & $\begin{array}{c}\text { Therm. } \\
\text { Cond. }\end{array}$ & $\begin{array}{c}\text { Rad. } \\
\text { Losses }\end{array}$ & Res. $^{a}$ \\
& & & & & & \\
\hline NN & 0 & $\infty$ & - & no & no & 132 \\
NR & 0 & $\infty$ & - & no & yes & 132 \\
TN & 0 & $\infty$ & - & yes & no & 132 \\
TR & 0 & $\infty$ & - & yes & yes & 132 \\
NN-Bx4 & 1.31 & 4 & $B_{\mathrm{x}}$ & no & no & 132 \\
NN-By4 & 1.31 & 4 & $B_{\mathrm{y}}$ & no & no & 132 \\
NN-Bz4 & 1.31 & 4 & $B_{\mathrm{z}}$ & no & no & 132 \\
TN-Bx4 & 1.31 & 4 & $B_{\mathrm{x}}$ & yes & no & 132 \\
TN-By4 & 1.31 & 4 & $B_{\mathrm{y}}$ & yes & no & 132 \\
TN-Bz4 & 1.31 & 4 & $B_{\mathrm{z}}$ & yes & no & 132 \\
TR-Bx1 & 2.63 & 1 & $B_{\mathrm{x}}$ & yes & yes & 132 \\
TR-By1 & 2.63 & 1 & $B_{\mathrm{y}}$ & yes & yes & 132 \\
TR-Bz1 & 2.63 & 1 & $B_{\mathrm{z}}$ & yes & yes & 132 \\
TR-Bx4 & 1.31 & 4 & $B_{\mathrm{x}}$ & yes & yes & 132 \\
TR-By4 & 1.31 & 4 & $B_{\mathrm{y}}$ & yes & yes & 132 \\
TR-Bz4 & 1.31 & 4 & $B_{\mathrm{z}}$ & yes & yes & 132 \\
TR-Bx100 & 0.26 & 100 & $B_{\mathrm{x}}$ & yes & yes & 132 \\
TR-By100 & 0.26 & 100 & $B_{\mathrm{y}}$ & yes & yes & 132 \\
TR-Bz100 & 0.26 & 100 & $B_{\mathrm{z}}$ & yes & yes & 132 \\
& & & & & & \\
TR-Bz4-hr & 1.31 & 4 & $B_{\mathrm{z}}$ & yes & yes & 264 \\
TR-Bz4-hr2 & 1.31 & 4 & $B_{\mathrm{z}}$ & yes & yes & 528 \\
& & & & & & \\
\hline
\end{tabular}

${ }^{\mathrm{a}}$ Initial number of zones per cloud radius

extending infinitely along the $z$ axis perpendicular to the $(x, y)$ plane. The primary shock propagates along the $y$ axis. In this geometry, we consider three different field orientations: 1) parallel to the planar shock and perpendicular to the cylindrical cloud, 2) perpendicular to both the shock front and the cloud, and 3) parallel to both the shock and the cloud. The magnetic field components along the $x$ and the $z$ axis are enhanced by a factor $(\gamma+1) /(\gamma-1)$ (where $\gamma$ is the ratio of specific heats) in the post-shock region (in the strong shock limit; Zel'dovich \& Raizer 1966), whereas the component along the $y$ axis is continuous across the shock. We include runs in the strong and weak magnetic field limits, considering initial field strengths of $|\mathbf{B}|=2.63,1.31,0.26,0 \mu \mathrm{G}$ in the unperturbed ambient medium ${ }^{7}$, corresponding to $\beta_{0}=1,4,100, \infty$, where $\beta_{0}=P /\left(B^{2} / 8 \pi\right)$ is the ratio of thermal to magnetic pressure in the pre-shock region. This range of $\beta_{0}$ includes typical values inferred for the diffuse regions of the ISM (e.g. Mac Low \& Klessen 2004) and for shock-cloud interaction regions in evolved SNR shells (e.g Bocchino et al. 2000). There is no magnetic field component exclusively associated to the cloud.

We follow the shock-cloud interaction for $3.5 \tau_{\mathrm{cc}}$, where $\tau_{\mathrm{cc}} \approx \chi^{1 / 2} r_{\mathrm{cl}} / w$ is the cloud crushing time, i.e. the characteristic time of the shock transmission through the cloud; for the conditions considered here $(\chi=10$ and $\mathcal{M}=50), \tau_{\mathrm{cc}} \approx 5.4 \times 10^{3} \mathrm{yr}$. Each simulation is repeated either with or without thermal conduction for each field orientation. Table 2 lists the runs and the initial physical parameters of the simulations.

We solve numerically the set of MHD equations us-

7 The unmagnetized case (i.e. $|\mathbf{B}|=0$ ) described here is analogous to the one studied in Paper I except for the fact that in the present case the cloud is a cylinder rather than a sphere and has smooth boundaries. 

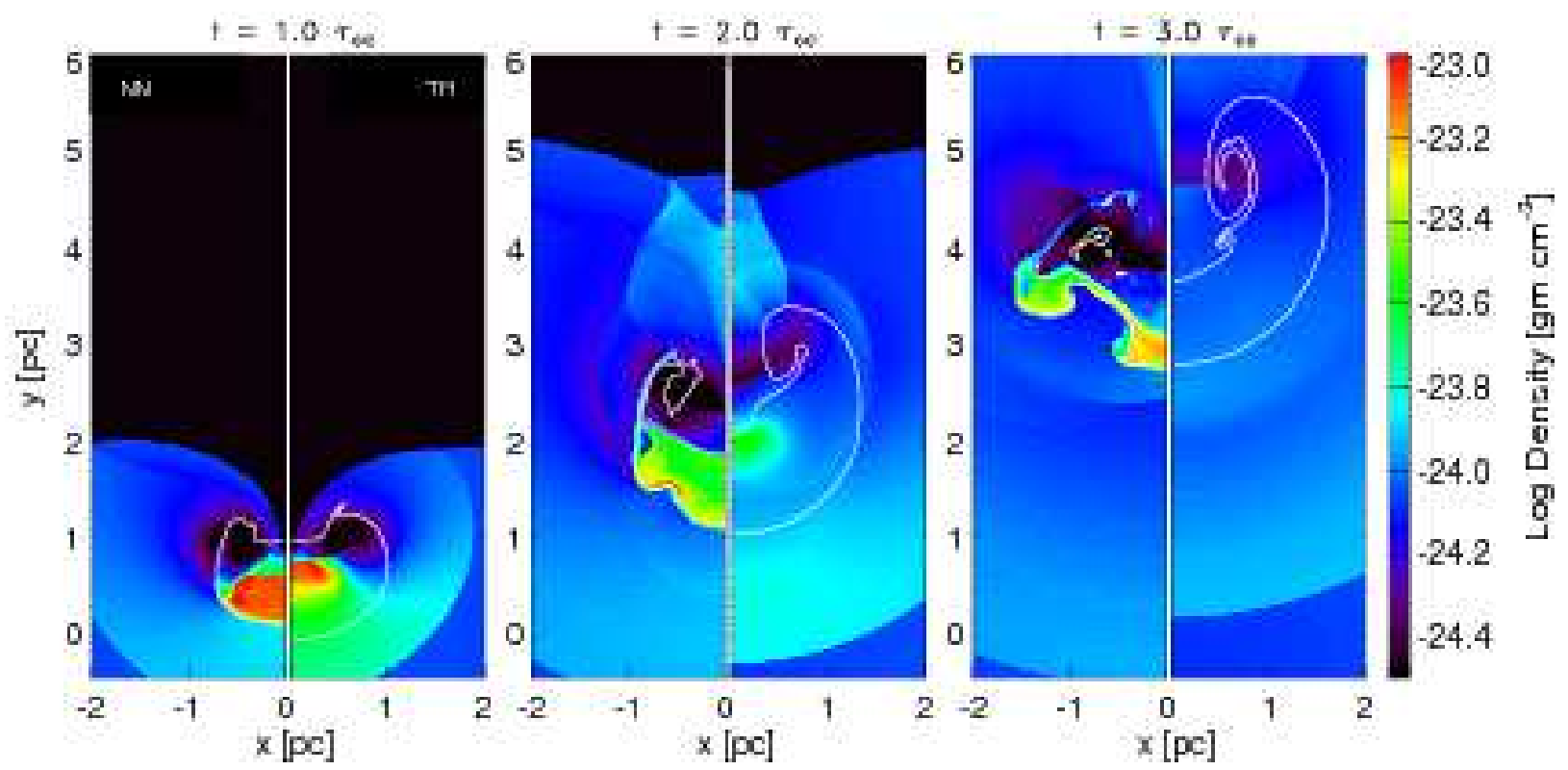

FIG. 1.- Mass density distribution $\left(\mathrm{gm} \mathrm{cm}^{-3}\right)$ in the $(x, y)$ plane, in log scale, in the simulations NN (left half panels) and TR (right half panels), sampled at the labeled times in units of $\tau_{\mathrm{cc}}$. The contour encloses the cloud material.

ing FLASH (Fryxell et al. 2000), a multiphysics code including the PARAMESH library (MacNeice et al. 2000) for the adaptive mesh refinement. The MHD equations are solved using the FLASH implementation of the HLLE scheme (Einfeldt 1988). The code has been extended with additional computational modules to handle the radiative losses and the anisotropic thermal conduction (see Pagano et al. 2007, for the details of the implementation).

The 2.5D Cartesian $(x, y)$ grid extends between -4 and $4 \mathrm{pc}$ in the $x$ direction and between -1.4 and $6.6 \mathrm{pc}$ in the $y$ direction. Initially the cloud is located at $(x, y)=(0,0)$ and the primary shock front propagates in the direction of the $y$ axis. At the coarsest resolution, the adaptive mesh algorithm used in the FLASH code uniformly covers the 2.5D computational domain with a mesh of $4^{2}$ blocks, each with $8^{2}$ cells. We allow for 5 levels of refinement, with resolution increasing twice at each refinement level. The refinement criterion adopted (Löhner 1987) follows the changes of the density and of the temperature. This grid configuration yields an effective resolution of $\approx 7.6 \times 10^{-3} \mathrm{pc}$ at the finest level, corresponding to $\approx 132$ cells per cloud radius. In Sect. 3.5, we discuss the effect of spatial resolution on our results, considering the additional runs TR-Bz4-hr and TR-Bz4-hr2 which use an identical setup to run TR-Bz4, but with higher resolution $(\approx 264$ and $\approx 528$ cells per cloud radius, respectively; see Table 2).

We use a constant inflow boundary condition for the post-shock gas at the lower boundary, with free outflow elsewhere. For runs with zero magnetic field $\left(\beta_{0}=\infty\right)$, we use reflecting boundary conditions at $x=0$ along the symmetry axis of the problem and only evolve half of the grid.

\section{RESULTS}

\subsection{Dynamical evolution}

Figs. 1 and 2 show the evolution of the mass density in the $(x, y)$ plane in the simulations with $\beta_{0}=\infty$ (runs NN and TR) and with $\beta_{0}=4$ (runs NN-Bx4, NN-By4, NN-
Bz4, TR-Bx4, TR-By4, TR-Bz4). The left (right) half panels show the result of models without (with) thermal conduction and radiative losses.

From Fig. 1, we note that the thermal conduction drives the cloud evolution in the unmagnetized case $\left(\beta_{0}=\infty\right.$; run TR): after the initial compression due to the primary shock, the cloud expands and gradually evaporates due to the heating driven by the thermal conduction in a few dynamical timescales (see right half panels in Fig. 1). The heat conduction strongly contrasts the radiative cooling of some parts of the cloud and no thermal and hydrodynamic instabilities (visible in run NN; see left-panels in Fig. 1) develops during the cloud evolution, making the cloud more stable and longer-living (the mass mixing is strongly reduced; see Paper I for more details).

We now discuss the effect of the magnetic-field-oriented (anisotropic) thermal conduction on the shock-cloud collision when an ambient magnetic field permeates the ISM. We first summarize the expected evolution in the presence of an ambient magnetic field, according to the well-established results of previous models without thermal conduction. We distinguish between fields perpendicular to the cylindrical clouds (i.e. with only $B_{\mathrm{x}}$ and $B_{\text {y }}$ components; referred to as "external" fields by Fragile et al. 2005) and fields parallel to the cylindrical clouds (i.e. with only the $B_{\mathrm{z}}$ component; referred to as "internal" fields). In the former case, the magnetic field plays a dominant role along the cloud surface and in the wake of the cloud where it reaches its highest strength (and the plasma $\beta$ its lowest values; e.g. Mac Low et al. 1994; Jones et al. 1996). In the case of $B_{\mathrm{x}}$, the magnetic field is trapped at the nose of the cloud, leading to a continuous increase of the magnetic pressure and field tension there (see upper panels in Fig. 2); in the case of $B_{\mathrm{y}}$, the cloud expansion leads to the increase of magnetic pressure and field tension laterally to the cloud (see middle panels in Fig. 2). In the case of $B_{\mathrm{z}}$ (internal field), the magnetic field, being parallel to the cylindrical cloud, modifies only the total effective pressure of the plasma 

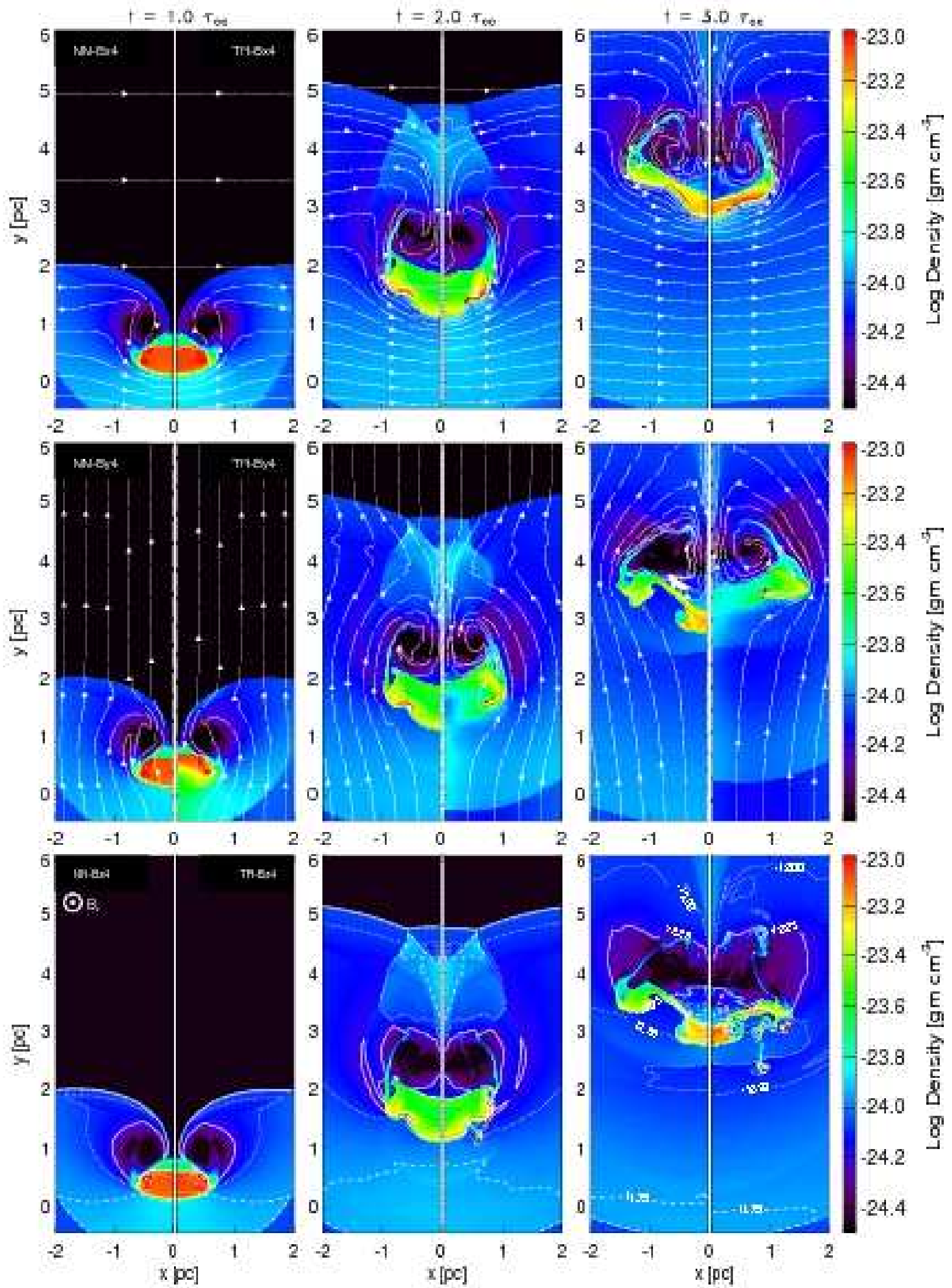

Fig. 2.- As in Fig. 1 for the simulations with $\beta_{0}=4$ and the magnetic field oriented along $x$ (upper panels), $y$ (middle panels), and $z$ (lower panels). The figure shows the distribution in models either without (left half panels) or with (right half panels) thermal conduction and radiative losses. For runs NN-Bx4, TR-Bx4, NN-By4 and TR-By4, we plot the magnetic field lines; for runs NN-Bz4 and TR-Bz4, we include contours of $\log \left(B^{2} / 8 \pi\right)$. 
(Jones et al. 1996); in the case of radiating shocks, the additional magnetic pressure may play a crucial role in the shocked cloud, preventing further compression of the cloud material (Fragile et al. 2005).

\subsubsection{External magnetic fields}

In the case of predominantly external magnetic fields, Mac Low et al. (1994) and Jones et al. (1996) have shown that the hydrodynamic instabilities can be suppressed even in models neglecting the thermal conduction due to the tension of the magnetic field lines which maintain a more laminar flow around the cloud surface (see also Fragile et al. 2005): for a $\gamma=5 / 3$ gas, the KH instabilities are suppressed if $\beta<2 / \mathcal{M}^{2}$, whereas RT instabilities are suppressed if $\beta<(2 / \gamma)(\chi / \mathcal{M})^{2}$ (see also Chandrasekhar 1961). However, for the parameters used in this paper $(\chi=10$ and $\mathcal{M}=50)$, the magnetic field cannot suppress $\mathrm{KH}$ instabilities in any of our runs, whereas the RT instabilities are suppressed only in runs that lead to locally very strong field $(\beta<0.05)$. This can be seen in model NN-Bx4 (upper panels in Fig. 2), presenting a large field increase at the cloud boundary, compared to model NN (Fig. 1): in the latter case the growth of $\mathrm{KH}$ and RT instabilities at the cloud boundary is much more evident than in NN-Bx4. On the other hand, the hydrodynamic instabilities are suppressed more efficiently in models including the thermal conduction (runs TR$\mathrm{Bx} 4$ and TR-By4) even in cases with low field increase (for instance in our $B_{\mathrm{y}}$ case) as it is evident in Fig. 2 by comparing models NN-Bx4 and NN-By4 with models TR-Bx4 and TR-By4, respectively.

The thermal exchanges between the cloud and the surrounding medium strongly depend on the initial field orientation. Fig. 3 shows the heat flux and magnetic field strength distributions in the $(x, y)$ plane in runs TRBx4, TR-By4, and TR-Bz4, at time $t=2 \tau_{\text {cc. }}$. In our $B_{\mathrm{x}}$ case (upper panels in Fig. 2), the magnetic field lines gradually envelope the cloud, reducing the heat conduction through the cloud surface (see left panels in Fig. 3): thermal exchanges between the cloud and the surrounding medium are channelled through small regions located at the side of the cloud. The cloud expansion and evaporation are strongly limited by the confining effect of the magnetic field (cf. the unmagnetized case TR in Fig. 1 with model TR-Bx4 in Fig. 2) that becomes up to 30 times stronger just outside the cloud than inside it (see, also, the lower left panel in Fig. 3). The consequent thermal insulation induces the radiative cooling and condensation of the plasma into the cloud during the phase of cloud compression $\left(t<\tau_{\mathrm{cc}}\right)$. At the end of this phase, the cloud material has temperature $T \approx 10^{5} \mathrm{~K}$ and density $n_{\mathrm{H}} \approx 10 \mathrm{~cm}^{-3}$ where primary and reverse shocks transmitted into the cloud are colliding; for these values of $T$ and $n_{\mathrm{H}}$, the Field length scale (Begelman \& McKee 1990) derived from the ratio of cooling timescale over conduction timescale (see Paper I for details) is

$$
l \approx 10^{6} \frac{T^{2}}{n_{\mathrm{H}}} \approx 3.2 \times 10^{-4} \mathrm{pc} .
$$

The radiative cooling dominates over the effects of the thermal conduction in cold and dense regions with dimensions larger than $l$. At variance with our unmagnetized case TR, therefore, thermal instabilities develop in run TR-Bx4. One of this cold and dense structures is evident in Fig. 2 (upper panels) and is located at the cloud boundary near the nose of the cloud (at $x \approx 0.4 \mathrm{pc}$ and $y \approx 3.0 \mathrm{pc})$ at $t=3 \tau_{\mathrm{cc}}$.

In the $B_{\mathrm{y}}$ case, the initial field direction is mostly maintained in the cloud core during the evolution, allowing efficient thermal exchange between the core and the hot medium upwind of the cloud (see center panels in Fig. 3): the core is gradually heated and evaporates in few dynamical timescales. This is illustrated by run TR-By4 in Fig. 2. On the other hand, the cloud is thermally insulated laterally where the magnetic field lines prevent thermal exchange between the cloud and the surrounding medium. Also, a strong magnetic field component along the $x$ axis develops in the wake of the cloud and inhibits thermal conduction with the medium downwind of the cloud. The thermal insulation at the side of the cloud determines the growth of thermal instabilities where shocks transmitted into the cloud collide (see middle panels in Fig. 2).

In both external field configurations, elongated structures of strong field concentration are produced on the axis downwind of the cloud due to the focalization of the magnetized fluid flows there (see upper and middle panels of Fig. 2, and lower panels in Fig. 3). These filamentary structures, identified as "flux ropes" by Mac Low et al. (1994), are formed by magnetic field lines stretched around the cloud shape and do not carry a significant amount of cloud material (as shown by the tracer $C_{\mathrm{cl}}$ ) although the plasma there moves with the cloud (see also Gregori et al. 2000).

\subsubsection{Internal magnetic fields}

Predominantly internal magnetic fields strongly suppress the heat conduction, providing an efficient thermal insulation of the cloud material (see right panels in Fig. 3). In the realistic configuration of an elongated cloud with finite length $L$ along the $z$ axis, some heat would be conducted along the magnetic field lines. The characteristic timescales for the conduction along magnetic field lines is (see Paper I)

$$
\tau_{\text {cond }} \approx 2.6 \times 10^{-9} \frac{n_{\mathrm{H}} L^{2}}{T^{5 / 2}} .
$$

We estimate that the cloud would thermalize in $\tau_{\text {cond }}>$ $3.5 \tau_{\mathrm{cc}}$ (i.e. the physical time covered by our simulations), if the length scale of the cloud along the $z$ axis is $L>3 \mathrm{pc}$. In this case, hydrodynamic instabilities develop at the cloud boundary, being both the magnetic field and the thermal conduction not able to suppress them. The growth of these instabilities is clearly seen in Fig. 2 (lower panels). The combined effect of hydrodynamic instabilities and shocks transmitted into the cloud leads to unstable high-density regions at the cloud boundaries that trigger the development of thermal instabilities there (see lower panels in Fig. 2). However, as discussed by Fragile et al. (2005), internal magnetic field lines are expected to resist compression in the shocked cloud, thus reducing the cooling efficiency. In fact, in our run TR-Bz4, the cloud material is prevented from cooling below $T \approx 10^{3} \mathrm{~K}$. Since the thermal conduction does not play any significant role in the shock-cloud interaction, our $B_{\mathrm{z}}$ case leads to results similar to those 

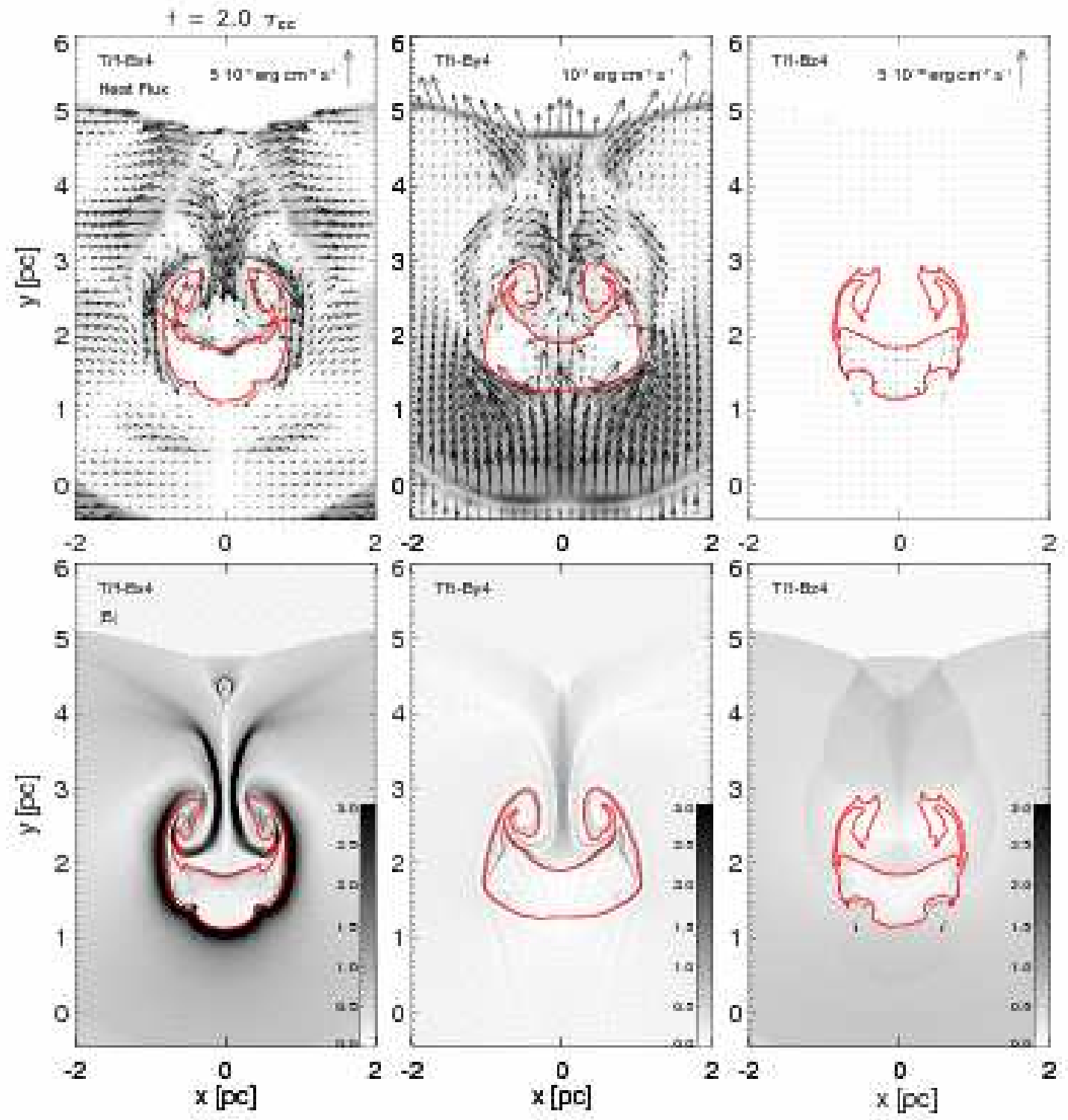

Fig. 3.- Heat flux (upper panels) and magnetic field strength (lower panels) distributions in the $(x, y)$ plane in the simulations TR-Bx4 (left panels), TR-By4 (center), and TR-Bz4 (right), at time $t=2 \tau_{\mathrm{cc}}$. The arrows in the upper panels describe the heat flux and scale linearly with respect to the reference value shown in the upper right corner of each panel. The scale of the magnetic field strength is linear and is given by the bar on the right, in units of $10 \mu \mathrm{G}$. The red contour encloses the cloud material.

obtained by Fragile et al. (2005) and we do not discuss further this case.

\subsection{Role of thermal conduction}

In this section, we study more quantitatively the effect of thermal conduction on the cloud evolution and, in particular, on the cloud compression and on the magnetic field increase. To this end, we use the tracer defined in Sect. 2 to identify zones whose content is the original cloud material by more than $90 \%$. Then, we define the cross-sectional area of cloud material, $A_{\mathrm{cl}}(t)$, as the total area in the $(x, y)$ plane occupied by these zones. We define the cloud compression (or expansion) as $A_{\mathrm{cl}} / A_{\mathrm{cl}}$, where $A_{\mathrm{cl} 0}$ is the initial cross-sectional area. We also define an average mass-weighted temperature of the cloud and an average magnetic field strength associated to the cloud as

$$
\begin{aligned}
\langle T\rangle_{\mathrm{cl}}= & \frac{\int_{A\left(C_{\mathrm{cl}}>0.9\right)} C_{\mathrm{cl}} \rho T d a}{\int_{A\left(C_{\mathrm{cl}}>0.9\right)} C_{\mathrm{cl}} \rho d a} \\
\langle B\rangle_{\mathrm{cl}}= & \frac{\int_{A\left(C_{\mathrm{cl}}>0.9\right)} C_{\mathrm{cl}} B d a}{\int_{A\left(C_{\mathrm{cl}}>0.9\right)} C_{\mathrm{cl}} d a}
\end{aligned}
$$

where we integrate on zones with $C_{\mathrm{cl}}>0.9$. Note that our choice of considering cells with the value of the passive tracer $C_{\mathrm{cl}}>0.9$ is arbitrary. To determine how sensitive the results are to this value and, in particular, to small changes in it, we derive our results also considering the values $C_{\mathrm{cl}}>0.85$ and $C_{\mathrm{cl}}>0.95$. In all the cases, we find that the results derived with the different thresholds show the same trend with differences lower than $10 \%$. 

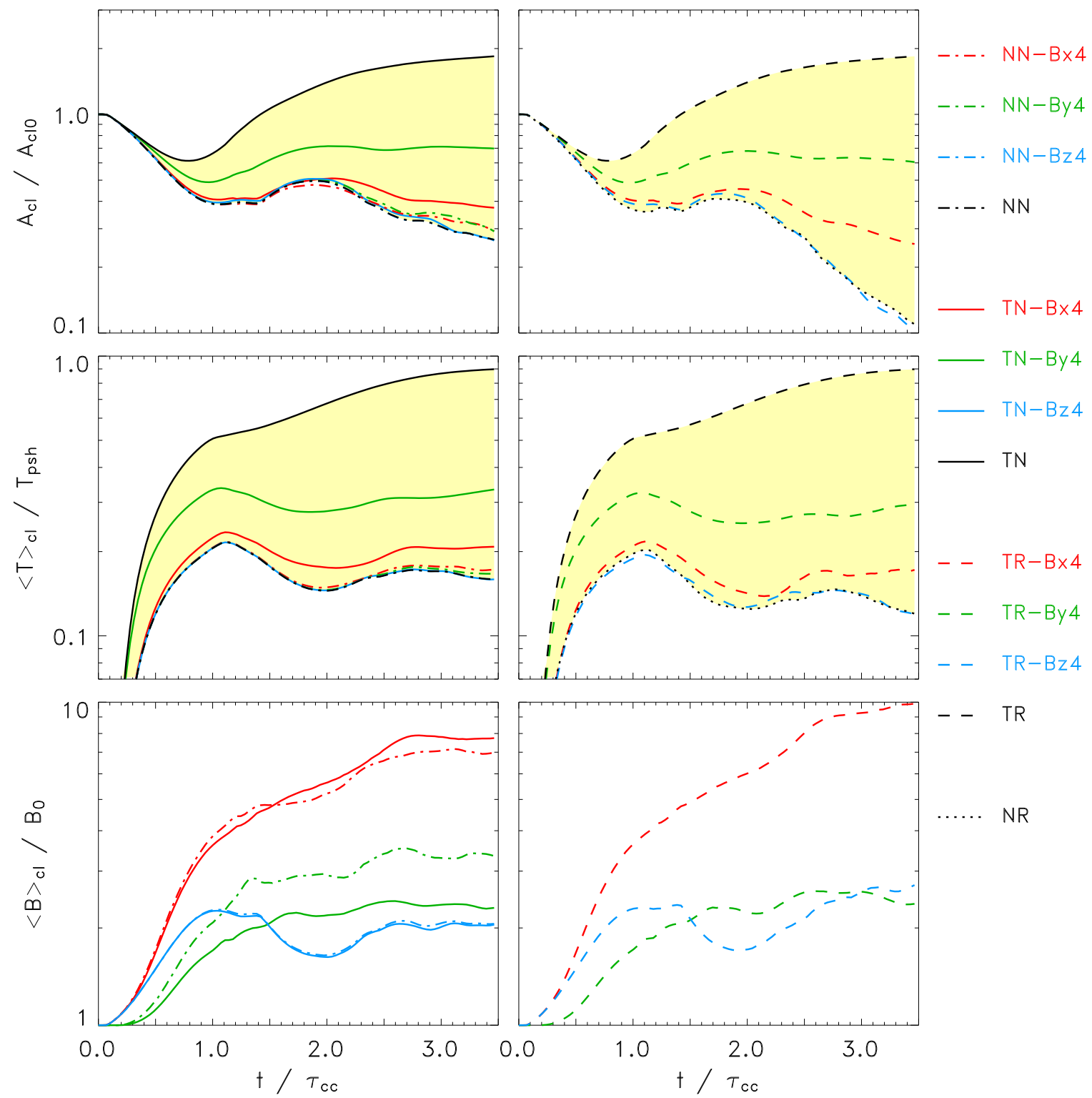

- $-\mathrm{TR}$

FIG. 4.- Evolution of cloud compression (upper panels), of average temperature (middle panels), and of average magnetic field strength (lower panels) of the cloud for runs which neglect the thermal conduction and the radiation (dot-dashed lines; left panels), for runs which include the thermal conduction but neglect the radiation (solid; left panels), for runs which include the radiation but neglect the thermal conduction (dotted; right panels) and for runs which include both physical effects (dashed; right panels). The magnetized cases with $\beta_{0}=4$ are marked with red (initial magnetic field along $x$ ), green (initial $\mathbf{B}$ along $y$ ) and blue (initial $\mathbf{B}$ along $z$ ) lines; the unmagnetized cases are marked with black lines. The light yellow regions mark the location of solutions which have thermodynamical characteristics in between the cases of maximum efficiency of the thermal conduction (models TN and TR) and the cases without thermal conduction (models NN and NR). By comparing the position of the magnetized models curves inside the yellow region, it is possible to quantitatively assess the degree of suppression of the effects of the thermal conduction by the magnetic fields.

Fig. 4 shows the cloud compression, $A_{\mathrm{cl}} / A_{\mathrm{cl} 0}$, the average temperature of the cloud, $\langle T\rangle_{\mathrm{cl}}$, normalized to the post-shock temperature of the surrounding medium $\left(T_{\mathrm{psh}}=4.7 \times 10^{6} \mathrm{~K}\right)$, and the average magnetic field strength associated to the cloud, $\langle B\rangle_{\mathrm{cl}}$, normalized to the initial field strength $\left(B_{0}=1.31 \mu \mathrm{G}\right.$, corresponding to $\left.\beta_{0}=4\right)$ as a function of time for models neglecting thermal conduction and radiation (hereafter NNs models), for models including conduction but neglecting radiation (TNs models), and for models including both conduction and radiation (TRs models); we also include the results derived from the unmagnetized case NR with radiative cooling and without thermal conduction. The figure shows both the magnetized cases with $\beta_{0}=4$ and the unmagnetized cases (see Table 2).

In all the NNs models either with (NN-Bx4, NN-By4, and NN-Bz4) or without (NN) the magnetic field, the evolution of the cloud compression and of the average cloud temperature is roughly the same (see left panels in Fig. 4). The cloud is initially compressed over a timescale $t \approx \tau_{\mathrm{cc}}$ due to the ambient post-shock pressure; during this phase $\langle T\rangle_{\mathrm{cl}}$ rapidly increases. After $t \approx \tau_{\mathrm{cc}}$, the cloud partially reexpands, leading to a decrease of $\langle T\rangle_{\mathrm{cl}}$. In the last phase $\left(t>2.0 \tau_{\mathrm{cc}}\right)$, the cloud is compressed again by the interaction with the "Mach stem" formed during the reflection of the primary shock at the symmetry axis, and $\langle T\rangle_{\mathrm{cl}}$ increases; later $A_{\mathrm{cl}} / A_{\mathrm{cl} 0}$ continues to decrease, because of the mixing of the cloud material 
with the ambient medium (see Sect. 3.3; see also Paper I), while $\langle T\rangle_{\mathrm{cl}}$ stabilizes at $\approx 0.17 T_{\mathrm{psh}}$.

The field increase in the cloud material depends on the initial configuration of $\mathbf{B}$ (see lower left panel in Fig. 4). In the case of external fields ( $B_{\mathrm{x}}$ and $B_{\mathrm{y}}$ components), B is mainly intensified due to stretching of field lines due to sheared motion. In the $B_{\mathrm{x}}$ case, the magnetic field undergoes the greatest increase and $\langle B\rangle_{\mathrm{cl}}$ keeps increasing during the whole evolution. In fact the field is mainly intensified at the nose of the cloud where the background flow continues to stretch the field lines during the evolution (see upper panels in Fig. 2). In the $B_{\text {y }}$ case, the field increase occurs mainly at the side of the cloud where the field lines are stretched along the cloud surface. In the case of internal fields ( $B_{\mathrm{z}}$ component), the field increase is due to squeezing of field lines through compression. $\langle B\rangle_{\mathrm{cl}}$, therefore, follows the changes in $A_{\mathrm{cl}} / A_{\mathrm{cl} 0}$, since the field is locked within the cloud material. Thus the greatest field increase occurs at $t \approx \tau_{\mathrm{cc}}$ when the shocks transmitted into the cloud collide.

The effects of thermal conduction are greatest in the unmagnetized model (TN) which can be considered an extreme limit case (see left panels in Fig. 4). During the first stage of evolution $\left(t<0.8 \tau_{\mathrm{cc}}\right)$, the cloud is heated efficiently by the thermal conduction and its average temperature increases rapidly to $\sim 0.5 T_{\mathrm{psh}}$. As a consequence, the pressure inside the cloud increases and the cloud reexpands earlier than in model NN. Afterwards, the average cloud temperature, $\langle T\rangle_{\mathrm{cl}}$, keeps increasing up to $\sim 0.9 T_{\mathrm{psh}}$ at $t=3.5 \tau_{\mathrm{cc}}$.

In the case of predominantly external magnetic fields (models TN-Bx4 and TN-By4), the thermal conduction still plays a significant role in the cloud evolution, although its effects are not as large as in the unmagnetized case $(\mathrm{TN})$. During the initial compression, the thermal conduction contributes to the cloud heating: the average temperature of the cloud reaches values larger than in models neglecting the conduction (compare TNs with NNs models in the left panels of Fig. 4). This effect is greatest in the $B_{\mathrm{y}}$ case which is the configuration of field lines that allows the most efficient thermal exchange between the cloud and the hot environment (see Sect. 3.1). At $t=3.5 \tau_{\mathrm{cc}},\langle T\rangle_{\mathrm{cl}}$ in TNs models reaches values larger than in NNs models $\left(\approx 0.21 T_{\mathrm{psh}}\right.$ in the $B_{\mathrm{x}}$ case and $\approx 0.33 T_{\mathrm{psh}}$ in the $B_{\mathrm{y}}$ case). For internal magnetic fields, the thermal conduction plays no role in the evolution of the shocked cloud, being strongly ineffective due to $\mathbf{B}$ (see Sect. 3.1). As a consequence, the TN-Bz4 model leads to the same results as NNs models.

In general, therefore, the effects of the thermal conduction in the presence of an ambient magnetic field are reduced with respect to the corresponding unmagnetized case, but not entirely suppressed. This can be seen in Fig. 4, where we have marked in light yellow the region between the fully conductive unmagnetized case (TN) and the case without thermal conduction (NN). The magnetized TNs models are always within this region, meaning that the effects of the thermal conduction are never as large as in the unmagnetized case (TN) but not completely suppressed as in the model NN.

We also note that the thermal conduction influences indirectly the magnetic field increase. The main changes are in the $B_{\mathrm{y}}$ case and are due to the larger expansion of the cloud that reduces the increase of the field associ- ated to the cloud, being the field locked within the cloud material.

In our unmagnetized case TR (including thermal conduction and radiative cooling), the thermal conduction prevents the onset of thermal instabilities, and the evolution of the shocked cloud is the same as found in the TN model. At variance with our unmagnetized case TR, Fig. 4 shows that thermal instabilities develop in all our magnetized TRs runs, being the effects of thermal conduction reduced by the magnetic field. The effects of radiative cooling are very strong for internal fields (our $B_{\mathrm{z}}$ case; see run TR-Bz4 in Fig. 4). In this case, the heat conduction is totally suppressed by the magnetic field and the evolution of $A_{\mathrm{cl}} / A_{\mathrm{clo}}$ and of $\langle T\rangle_{\mathrm{cl}}$ are the same as those found in the unmagnetized case with radiative cooling and without thermal conduction (model $\mathrm{NR}$ ); at $t=3.5 \tau_{\mathrm{cc}}$, run TR-Bz4 (and NR) shows the largest cloud compression $\left(A_{\mathrm{cl}} / A_{\mathrm{cl} 0} \approx 0.1\right)$ and the lowest cloud average temperature $\left(\langle T\rangle_{\mathrm{cl}} \approx 0.12 T_{\mathrm{psh}}\right)$. In the case of external fields (runs TR-Bx4 and TR-By4), the effects of heat conduction are reduced but not suppressed and the results are intermediate between those derived for runs NR and TR (i.e. within the light yellow region in the right panels in Fig. 4). The cooling efficiency is largely reduced in our $B_{\mathrm{y}}$ case (run TR-By4), namely that with the magnetic field configuration that allows the most effective thermal conduction.

\subsection{Mass mixing and energy exchange}

We use the tracer to derive the cloud mass, $M_{\mathrm{cl}}$, as the total mass in zones whose content is the original cloud material by more than $90 \%$,

$$
M_{\mathrm{cl}}=L \int_{A\left(C_{\mathrm{cl}}>0.9\right)} C_{\mathrm{cl}} \rho d a,
$$

where $L$ is the cloud length along the $z$ axis, and the integral is done on zones with $C_{\mathrm{cl}}>0.9$. We investigate the mixing of cloud material with the ambient medium by defining the remaining cloud mass as $M_{\mathrm{cl}} / M_{\mathrm{cl} 0}$, where $M_{\text {clo }}$ is the initial cloud mass.

The tracer allows us to investigate also the energy exchange between the cloud and the surrounding medium; we derive the internal energy, $\mathcal{I}_{\mathrm{cl}}$, and the kinetic energy, $\mathcal{K}_{\mathrm{cl}}$ of the cloud as

$$
\begin{gathered}
\mathcal{I}_{\mathrm{cl}}=L \int_{A\left(C_{\mathrm{cl}}>0.9\right)} C_{\mathrm{cl}} \rho \epsilon d a, \\
\mathcal{K}_{\mathrm{cl}}=\frac{L}{2} \int_{A\left(C_{\mathrm{cl}}>0.9\right)} C_{\mathrm{cl}} \rho|\mathbf{u}|^{2} d a,
\end{gathered}
$$

where again $L$ is the cloud length along the $z$ axis, and the integral is done on zones with $C_{\mathrm{cl}}>0.9$. We also define the total energy of the cloud as

$$
E_{\mathrm{cl}}=\mathcal{I}_{\mathrm{cl}}+\mathcal{K}_{\mathrm{cl}}
$$

Fig. 5 shows the evolution of the cloud mass, $M_{\mathrm{cl}} / M_{\mathrm{cl0}}$, for NNs, TNs, and TRs models; again we also include the unmagnetized case with radiative cooling and without thermal conduction (model NR). Both unmagnetized cases and magnetized cases with $\beta_{0}=4$ are shown. In models without thermal conduction and radiation (NNs models), the hydrodynamic instabilities drive the mass 

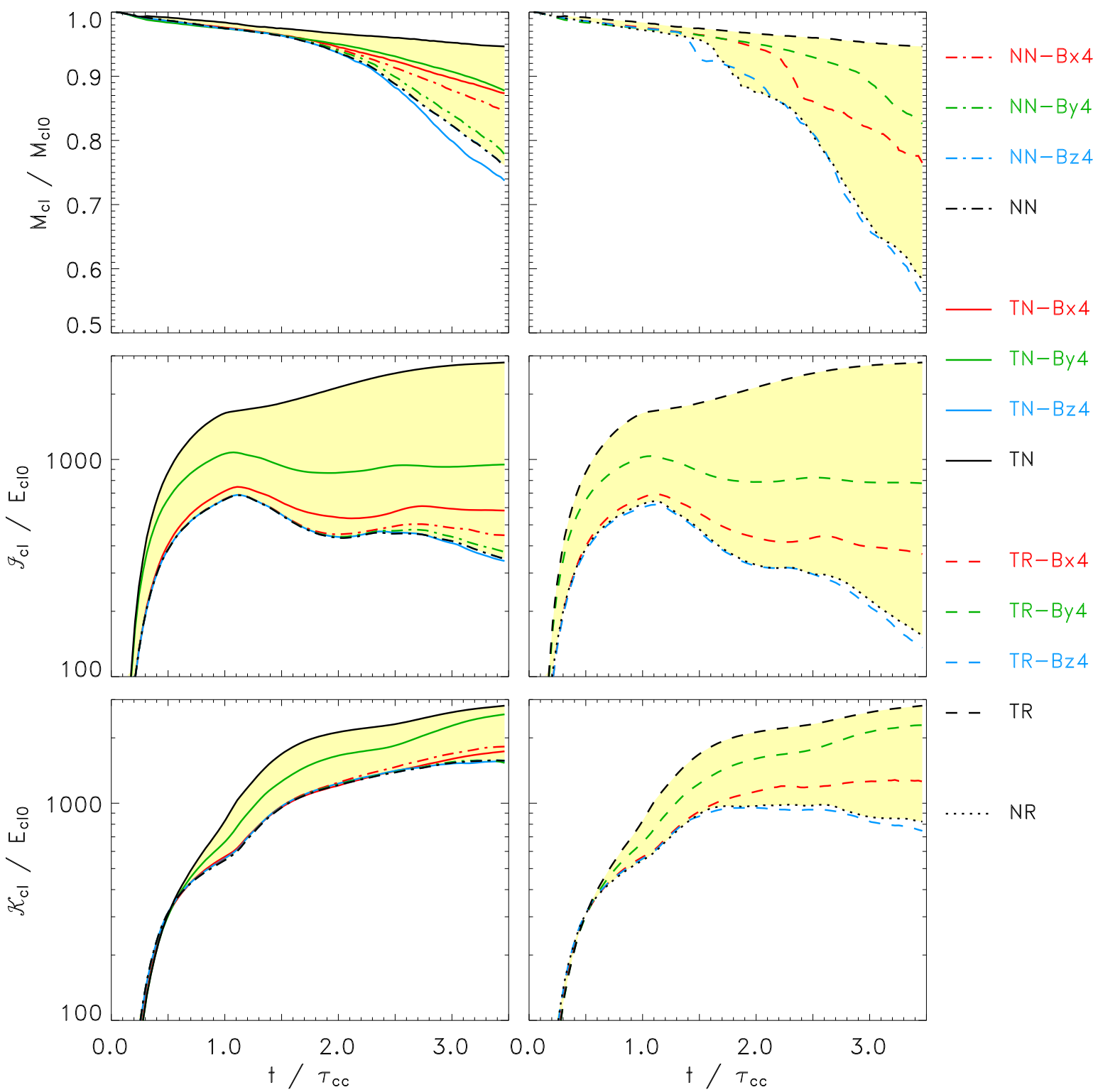

Fig. 5.- Presentation as in Fig. 4 for the evolution of the cloud mass (upper panels), of the internal energy of the cloud (middle panels), and of the kinetic energy of the cloud (lower panels).

mixing of the cloud ${ }^{8}$. The mass loss rate of the cloud, $\dot{m}_{\mathrm{cl}}$, increases significantly after $1.5 \tau_{\mathrm{cc}}$ (i.e. after the hydrodynamic instabilities have fully developed at the cloud boundary), with $\dot{m}_{\mathrm{cl}} \approx 1.5 \times 10^{-6} L_{\mathrm{pc}} M_{\odot} \mathrm{yr}^{-1}$, where $L_{\mathrm{pc}}$ is the cloud length along the $z$ axis in units of pc: $\sim 20 \%$ of the cloud mass is contained in mixed zones at $t=3.5 \tau_{\mathrm{cc}}$. The only exception is run NN-Bx4 $(\sim 15 \%$ of the cloud mass is in mixed zones at $\left.t=3.5 \tau_{\mathrm{cc}}\right)$, being in this case RT instabilities partially suppressed by the magnetic field (compare run NN-Bx4 with runs NN-By4 and NN-Bz4 in Fig. 2).

In TNs models with external magnetic fields (TN$\mathrm{Bx} 4$ and $\mathrm{TN}-\mathrm{By} 4)$, the mass loss rate of the cloud is less efficient than in NNs models with $\dot{m}_{\mathrm{cl}} \approx 6 \times$ $10^{-7} L_{\mathrm{pc}} \quad M_{\odot} \mathrm{yr}^{-1}(\sim 10 \%$ of the cloud mass is in mixed zones at $\left.t=3.5 \tau_{\mathrm{cc}}\right)$. In fact, in these cases the thermal

\footnotetext{
8 This is also true in our magnetized cases because, for the parameters used in this paper $(\mathcal{M}=50$ and $\chi=10)$, the hydrodynamic instabilities are partially suppressed by the magnetic field only in runs evolving to strong fields (see Sect. 3.1).
}

conduction suppresses most of the hydrodynamic instabilities and the mass loss mainly comes from the cloud evaporation driven by the thermal conduction rather than from hydrodynamic ablation. Note that our unmagnetized TN model is an extreme limit case in which the hydrodynamic instabilities are totally suppressed by the thermal conduction which drives the cloud mixing; in this case, the mass loss rate is $\dot{m}_{\mathrm{cl}} \approx 1.5 \times 10^{-7} L_{\mathrm{pc}} M_{\odot} \mathrm{yr}^{-1}$ ( $\sim 5 \%$ of the cloud mass is in mixed zones at $t=3.5 \tau_{\mathrm{cc}}$ ).

In magnetized TRs models, the onset of thermal instabilities increases the mass loss rate of the cloud with respect to the unmagnetized case $\left(\dot{m}_{\mathrm{cl}}\right.$ ranges between $1.5 \times 10^{-6} L_{\mathrm{pc}} \quad M_{\odot} \mathrm{yr}^{-1}$ and $\left.4 \times 10^{-6} L_{\mathrm{pc}} M_{\odot} \mathrm{yr}^{-1}\right)$ due to the fragmentation of the cloud in dense and cold cloudlets. We expect, therefore, that the larger the amount of cloud mass mixed with the surrounding medium at the end of the evolution, the more limited the thermal exchange between the cloud and the hot ambient medium (and, therefore, the greater the efficiency of radiative cooling). In fact, the upper right panel in Fig. 5 
shows that the mass mixing has the greatest efficiency in run TR-Bz4 (i.e. in the case with the thermal conduction totally suppressed) which shows a mass loss rate of the cloud similar to that derived from the unmagnetized NR model. On the other hand, in runs TR-Bx4 and TR-By4, the mass mixing is intermediate between those derived with runs NR and TR.

Fig. 5 also shows the evolution of internal (middle panels) and kinetic (lower panels) energy of the cloud, normalized to the initial total energy of the cloud, $E_{\mathrm{cl0}}$. Among the magnetized cases considered, the greatest values of $\mathcal{I}_{\mathrm{cl}}$ are reached in our $B_{\mathrm{y}}$ case which is the field configuration that allows the most efficient thermal exchange between the cloud and the environment; the increase of $\mathcal{I}_{\mathrm{cl}}$ is due to the heat conducted to the shocked cloud. Also, the $B_{\mathrm{y}}$ case leads to the greatest values of $\mathcal{K}_{\mathrm{cl}}$ because the cloud has a larger cross-sectional area (because of the larger cloud expansion due to the heating driven by heat conduction; see upper panels in Fig. 4) and offers, therefore, a larger surface to the pressure of the shock front responsible of the cloud acceleration.

\subsection{Role of the initial field strength}

In this section we explore the effects of the initial field strength on the mass mixing and energy exchange of the cloud. Fig. 6 shows the evolution of the cloud mass, $M_{\mathrm{cl}} / M_{\mathrm{cl} 0}$ (upper panel), and of the total (internal plus kinetic) energy of the cloud, $E_{\mathrm{cl}} / E_{\mathrm{cl} 0}$ (lower panel), for magnetized TRs models with different values of $\beta_{0}$. We discuss here only the cases of predominantly external magnetic fields ( $B_{\mathrm{x}}$ or $B_{\mathrm{y}}$ case) since no significant dependence on the initial field strength has been found in the case of predominantly internal magnetic fields $\left(B_{\mathrm{z}}\right.$ case).

Fig. 6 shows that the initial field strength plays a significant role in the $B_{\mathrm{x}}$ case. In particular, models with greater values of $\beta_{0}$ show a more efficient mixing of the cloud material and a less rapid increase of the cloud energy. As discussed in Sect. 3.3, in the $B_{\mathrm{x}}$ case, the rate of mass-loss from the cloud is mainly driven by ablation through the hydrodynamic instabilities (being the thermal conduction strongly suppressed by the magnetic field). On the other hand, in the case of external fields, the instabilities can be dumped by the magnetic field, depending on its strength (see Sect. 3.1.1). For instance, in the $B_{\mathrm{x}}$ case with $\beta_{0}=4$, we found that the RT instabilities are mostly suppressed by the magnetic field (see upper panels in Fig. 2). In the $B_{\mathrm{x}}$ case with $\beta_{0}=100$, instead the magnetic field is too weak to dump the hydrodynamic instabilities over the timescales considered; these instabilities, in turn, lead to the formation of regions dominated by the radiative cooling, triggering the development of thermal instabilities. Both the hydrodynamic and the thermal instabilities determine the cloud mass mixing (which is higher for higher values of $\beta_{0}$ ). In addition, the thermal instabilities reduce the increase of the cloud energy (which is less rapid for higher $\beta_{0}$ ) due to significant radiative losses.

In the $B_{\mathrm{y}}$ case, the initial field strength has a smaller influence on the dynamic and thermal evolution of the cloud than in the $B_{\mathrm{x}}$ case (see Fig. 6). In addition, at variance with the $B_{\mathrm{x}}$ case, models with greater values of $\beta_{0}$ show a less efficient mixing of the cloud material and a more rapid increase of the cloud energy. In the $B_{\mathrm{y}}$
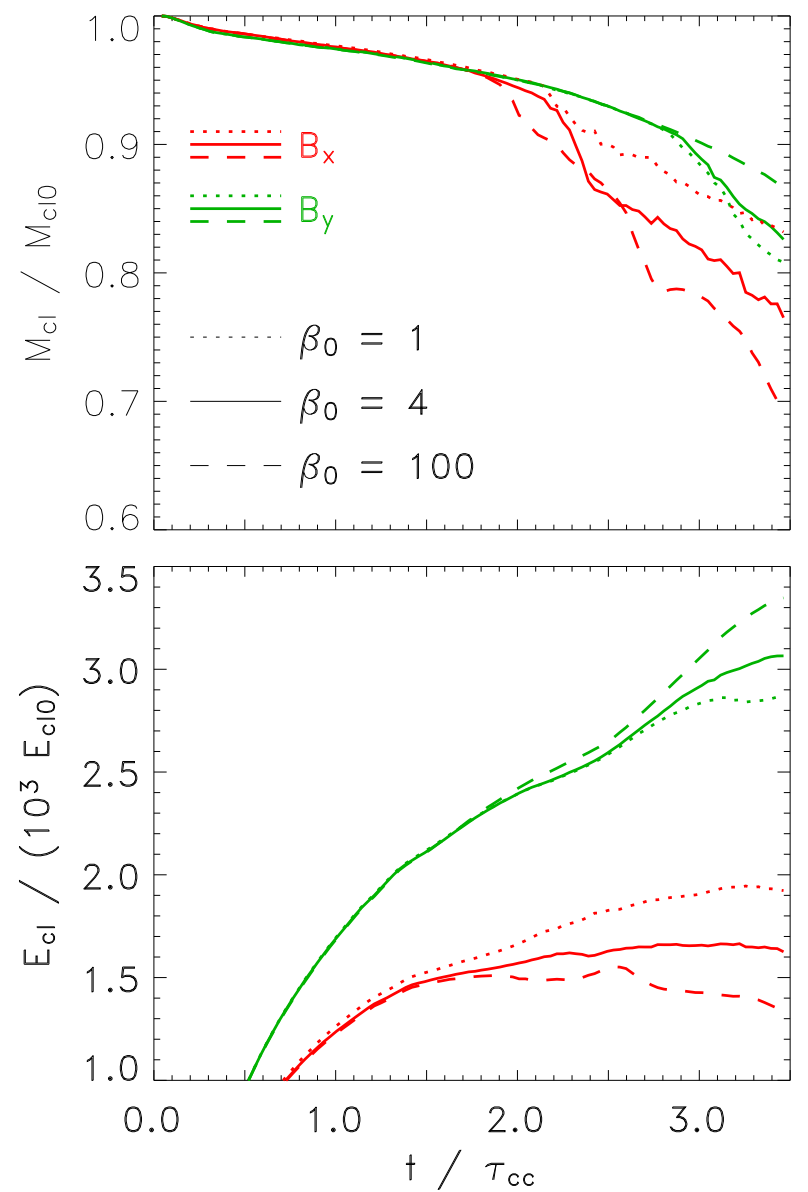

FIG. 6.- Evolution of the cloud mass (upper panel) and of the total energy of the cloud (internal plus kinetic; lower panel) for runs including both the thermal conduction and the radiative cooling (TRs models). The figure shows the simulations with the magnetic field oriented along $x$ (red lines) or $y$ (green) and with $\beta_{0}=1$ (dotted lines), 4 (solid), and 100 (dashed).

case, in fact, the hydrodynamic instabilities responsible of the mass mixing are mainly suppressed by the thermal conduction rather than by the magnetic field as in the $B_{\mathrm{x}}$ case. As a consequence, the higher the value of $\beta_{0}$, the more effective the thermal conduction in suppressing the instabilities and in heating the plasma, the less efficient the cloud mass mixing and the more rapid the increase of the cloud energy.

\subsection{Effect of spatial resolution}

The effective resolution adopted in our simulations is $\approx 132$ cells per cloud radius, a value above the resolution requirements suggested by Klein et al. (1994) for non-radiative clouds. However, for radiative clouds, we expect that the details of the plasma radiative cooling depend on the numerical resolution: a higher resolution may lead to different peak density and hence influence the cooling efficiency of the gas, preventing further compression of the cloud. In the non-conducting regime, Fragile et al. (2005) found that the results generally converge for simulations with resolution larger than 100 cells per cloud radius ( $\lesssim 10 \%$ differences). In the simulations presented here, the thermal conduction partially contrasts the radiative cooling in the case of external fields $\left(B_{\mathrm{x}}\right.$ or $\left.B_{\mathrm{y}}\right)$, alleviating the problem of numerical resolution (see also Paper I). 

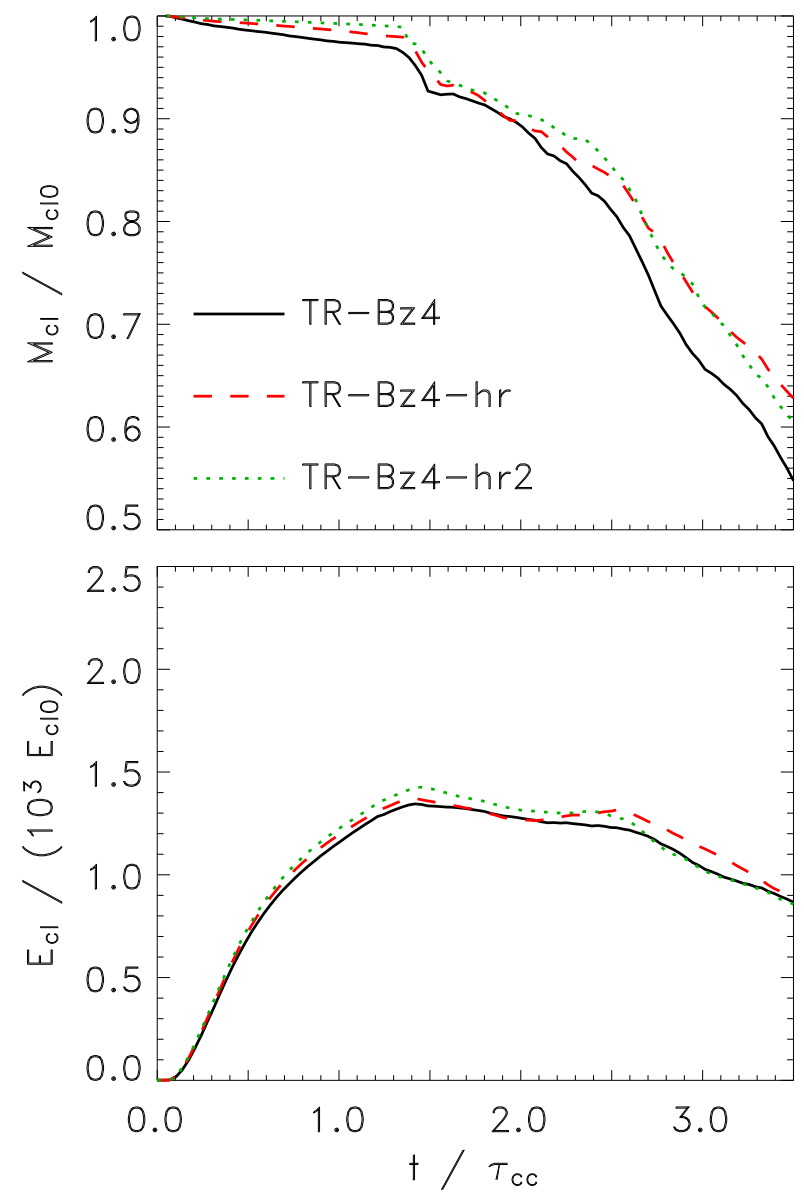

FIG. 7.- Presentation as in Fig. 6 for runs TR-Bz4 (solid lines), TR-Bz4-hr (dashed), and TR-Bz4-hr2 (dotted).

In order to check if our adopted resolution is sufficient to capture the basic cloud evolution over the time interval considered, we compare three simulations (TR-Bz4, TR-Bz4-hr, and TR-Bz4-hr2) with different spatial resolution (132, 264, and 528 zones per cloud radius, respectively) for the $B_{\mathrm{z}}$ case with $\beta=4$, namely one of the cases in which the growth of hydrodynamic and thermal instabilities is most prominent and the effect of thermal conduction (contrasting the development of hydrodynamic and thermal instabilities) is negligible. Since this case is one of the most demanding for resolution, it can be considered a worst case comparison of convergence.

Figure 7 compares the evolution of the cloud mass, $M_{\mathrm{cl}} / M_{\mathrm{cl} 0}$, and of the total energy of the cloud, $E_{\mathrm{cl}} / E_{\mathrm{cl} 0}$, for the three simulations TR-Bz4, TR-Bz4-hr, and TRBz4-hr2. In general, we find that the results obtained with the three simulations agree quite well in their qualitative behavior, showing differences $\lesssim 10 \%$. In runs TRBz4-hr and TR-Bz4-hr2, the remaining cloud mass and the total energy of the cloud are, in general, systematically higher than in run TR-Bz4. The larger mass mixing in TR-Bz4 is driven by the higher diffusion of the lowresolution grid down to the very small structures which tend to smear out concentrated density peaks, promoting mass mixing. The slightly lower energy of the cloud in TR-Bz4 is a consequence of the larger mass mixing derived in this run with respect to TR-Bz4-hr and TRBz4-hr2. Note that, in runs showing the onset of thermal instabilities (i.e NRs and TRs models), the size of the latters reaches the resolution limit toward the end of the simulations when the relevant physical processes are already at a late stage.

\section{SUMMARY AND CONCLUSION}

We investigated the importance of magnetic-fieldoriented thermal conduction in the interaction between an isolated elongated dense cloud and an interstellar shock-wave of an evolved SNR shell through numerical MHD simulations. To our knowledge, these simulations represent the first attempt to model the shock-cloud interaction that simultaneously considers magnetic fields, radiative cooling, and anisotropic thermal conduction. Our findings lead to several conclusions:

1. In general, we found that the effects of thermal conduction on the evolution of the shocked cloud are reduced in the presence of an ambient magnetic field with respect to the unmagnetized cases investigated in Paper I. The efficiency of anisotropic thermal conduction strongly depends on the initial magnetic field orientation and configuration. This efficiency is the largest when the initial $\mathbf{B}$ is aligned with the direction of propagation of the shock front, and is the smallest when $\mathbf{B}$ is aligned with the cylindrical cloud, namely when the heat conduction is completely suppressed by the magnetic field.

2. We found that the hydrodynamic instabilities are suppressed efficiently by the anisotropic thermal conduction when the initial magnetic field is perpendicular to the cylindrical cloud (a configuration referred to as "external fields"). On the contrary, in the case of $\mathbf{B}$ parallel to the cylindrical axis of the cloud (i.e. when the field has component only along the $z$ axis - internal field), hydrodynamic instabilities develop at the cloud boundary. We found that, for the parameters of the simulations chosen, the magnetic tension is unable to suppress alone the hydrodynamic instabilities.

3. As for thermal instabilities, we found that, depending on the magnetic field orientation, the heat flux contributes to the heating of some parts of the cloud, reducing the efficiency of radiative cooling there, and preventing any thermal instability.

4. The mass loss of the cloud due to mixing with the surrounding medium is mainly driven by hydrodynamic instabilities; in the case of external fields (initial $\mathbf{B}$ perpendicular to the cylindrical cloud) the anisotropic thermal conduction reduces the mass mixing of the cloud. In any case, the mass loss rate is larger than that in the corresponding unmagnetized case $\left(\dot{m}_{\mathrm{cl}} \approx 1.5 \times 10^{-7} L_{\mathrm{pc}} M_{\odot} \mathrm{yr}^{-1}\right.$, i.e. $\sim 5 \%$ of the cloud mass is in mixed zones at $\left.t=3.5 \tau_{\mathrm{cc}}\right)$, but can get very high when the thermal conduction is completely suppressed $\left(\dot{m}_{\mathrm{cl}} \approx 4 \times 10^{-6} L_{\mathrm{pc}} \quad M_{\odot} \mathrm{yr}^{-1}\right.$, i.e. $\sim 45 \%$ of the cloud mass is in mixed zones at $t=3.5 \tau_{\mathrm{cc}}$ ).

5. The thermal conduction mostly rules the energy exchange between the cloud and surrounding 
medium. The exchange is favored when the magnetic field configuration is such that the conductive flow is not suppressed (i.e. external field configurations, $B_{\mathrm{x}}$ and $B_{\mathrm{y}}$ cases), but it is never as high as in the absence of magnetic field. In the $B_{\mathrm{y}}$ case, the cloud core is efficiently heated and evaporates in few dynamical timescales.

6. In general, the initial magnetic field strength has a small influence on the dynamic and thermal evolution of the shocked cloud for the ranges of values explored in this paper (namely $0.26 \mu \mathrm{G} \leq|\mathbf{B}| \leq$ $2.63 \mu \mathrm{G})$.

It is worth noting that some details of our simulations depend on the choice of the model parameters. For instance, the onset of thermal instabilities or the evaporation of the whole cloud depends on the initial shock Mach number, and on the density and dimensions of the cloud. The cases that we present here (i.e. $\mathcal{M}=50, \chi=10$, and different configurations of $\mathbf{B}$ ) are representative of a regime in which both the thermal conduction and the radiative cooling play an important role in the evolution of the shocked cloud. Nevertheless, our analysis proves that anisotropic thermal conduction can not be neglected in investigations of the evolution of shocked interstellar clouds.

In our simulations, we consider laminar thermal conduction, although regions of strong turbulence of different strength and extent develop in the system (for instance, at the shear layers along the cloud boundary or at the vortex sheets in the cloud wake). In fact, the turbulence in these regions may have a significant effect on thermal conduction, leading to significant deviations of thermal conductivity from its laminar values (e.g. Narayan \& Medvedev 2001; Lazarian 2006); in some cases, the turbulence may enhance the heat transfer, exceeding the classical Spitzer value (Lazarian 2006). As a result, thermal conduction may be not only anisotropic (in the presence of the magnetic field) but also "inhomogeneous" due to the presence of turbulence. However, even modeling accurately the turbulent thermal conductivity, we do not expect significant changes in the results of our $B_{\mathrm{z}}$ case, being the thermal conduction strongly ineffective in the whole spatial domain; in the remaining cases $\left(B_{\mathrm{x}}\right.$ and $\left.B_{\mathrm{y}}\right)$, our modeled thermal conductivity could be underestimated in regions of strong turbulence, affecting some details of the simulations but not the main conclusion of the paper that, in general, anisotropic thermal conduction can play an important role in the evolution of the shocked cloud.

Note also that the field configurations studied in this work are highly idealized. More realistic fields are expected to have more complex topologies and, often, the field can be tangled and chaotic. In the latter case, the thermal conduction will approach isotropy, whereas the effect of MHD turbulence is expected to partially suppress the heat transfer within a factor $\sim 5$ below the classical Spitzer estimate ${ }^{9}$ (Narayan \& Medvedev 2001; Lazarian 2006). The shock-cloud collision in the presence of an organized ambient magnetic field, discussed here,

\footnotetext{
9 As already discussed, the MHD turbulence can even enhance
} the heat transfer in some cases (see Lazarian 2006). and that in the absence of magnetic field can be considered as extreme cases: the former leading to highly anisotropic thermal conduction, the latter to the classical Spitzer thermal conduction. The case of chaotic magnetic field is expected to fall in between these two.

Our simulations were carried out in 2.5D Cartesian geometry, implying that the modeled clouds are elongated along the $z$ axis. This choice is expected to affect some details of the simulations but not our main conclusions. Adopting a 3D Cartesian geometry and modeling a spherical cloud, the highly symmetric shock transmitted into the cloud converging on the symmetry axis would lead to compression stronger than those found in our 2.5D simulations, enhancing the radiative cooling. Also, 3D simulations would provide an additional degree of freedom for hydrodynamic instabilities, increasing the mass loss rate of the cloud in the cases in which the mass mixing of cloud material is driven by instabilities. Note that, for a spherical cloud, our $B_{\mathrm{x}}$ and $B_{\mathrm{z}}$ cases no longer differ.

Finally we assume, in our simulations, that the cloud and the ambient material have the same composition, implying that microscopic mass mixing due to shear instabilities would be irrelevant. In a more realistic condition, a cold dense cloud may have a different composition from the hot ambient flow and the degree of microscopic mixing may translate into different spectral signatures of the system. In this case, species diffusion could also be important, along with thermal conduction, to determine the degree of microscopic mixing of the materials and, consequently, one would have to ask about the typical values of the Lewis number (i.e. the ratio of thermal diffusivity to mass diffusivity) in the system.

It is worth emphasizing that the quantitative results of our simulations depend on the physical parameters of the model (shock Mach number, density contrast and dimension of the cloud, etc.) as well as on the basic assumptions of the model (geometry of the cloud, geometry of the ambient magnetic field, laminar thermal conduction, composition of the cloud and of the ambient medium, etc.). Nevertheless, our results undoubtedly show that the magnetic-field-oriented thermal conduction can play an important role in the evolution of the shock-cloud interaction (which depends on the magnetic field orientation and configuration) and, in particular, in the mass and energy exchange between the cloud and the hot surrounding medium. We conclude, therefore, that a self-consistent and quantitative description of the interaction between magnetized shock-waves and interstellar gas clouds should include the effects of thermal conduction.

The results presented here are interesting for the study of middle-aged SNR shells expanding into a magnetized ISM and whose morphology is affected by ISM inhomogeneities (for instance, G272.2-3.2, e.g. Egger et al. 1996; Cygnus Loop, e.g. Patnaude et al. 2002; Vela SNR, e.g. Miceli et al. 2005). It will be further interesting to extend the present study, by modeling the shock-cloud interaction in $3 \mathrm{D}$ with radiative cooling, anisotropic thermal conduction, and magnetic field included and, even, considering detailed comparisons of model results with observations. 
The authors thank Timur Linde for his help with the MHD portion of FLASH and the referee for constructive and helpful criticism. The software used in this work was in part developed by the DOE-supported ASC / Alliance Center for Astrophysical Thermonuclear Flashes at the University of Chicago, using modules for thermal conduction and optically thin radiation built at the Osservatorio Astronomico di Palermo. Most of the simulations have been executed at CINECA (Bologna, Italy) in the framework of the INAF-CINECA agreement on "High Performance Computing resources for Astronomy and Astrophysics". This work makes use of results produced by the PI2S2 Project managed by the Consorzio COMETA, a project co-funded by the Italian Ministry of University and Research (MIUR) within the Piano Operativo Nazionale "Ricerca Scientifica, Sviluppo Tecnologico, Alta Formazione" (PON 2000-2006); more information is available at http://www.pi2s2.it and http://www.consorzio-cometa.it. This work was supported in part by Istituto Nazionale di Astrofisica.

\section{REFERENCES}

Balbus, S. A. 1986, ApJ, 304, 787

Begelman, M. C., \& McKee, C. F. 1990, ApJ, 358, 375

Bocchino, F., Maggio, A., Sciortino, S., \& Raymond, J. 2000, A\&A, 359,316

Borkowski, K. J., Shull, J. M., \& McKee, C. F. 1989, ApJ, 336, 979

Chandrasekhar, S. 1961, Hydrodynamic and hydromagnetic stability (International Series of Monographs on Physics, Oxford: Clarendon, 1961)

Cowie, L. L., \& McKee, C. F. 1977, ApJ, 211, 135

Egger, R., Greiner, J., \& Aschenbach, B. 1996, in Roentgenstrahlung from the Universe, MPE Report 263, 247-248

Einfeldt, B. 1988, SIAM J. Numer.Anal, 25, 357

Fadeyev, Y. A., Le Coroller, H., \& Gillet, D. 2002, A\&A, 392, 735

Fragile, P. C., Anninos, P., Gustafson, K., \& Murray, S. D. 2005, ApJ, 619, 327

Fragile, P. C., Murray, S. D., Anninos, P., \& van Breugel, W. 2004, ApJ, 604, 74

Fryxell, B. et al. 2000, ApJS, 131, 273

Giuliani, J. L. 1984, ApJ, 277, 605

Gregori, G., Miniati, F., Ryu, D., \& Jones, T. W. 2000, ApJ, 543, 775

Jones, T. W., Ryu, D., \& Tregillis, I. L. 1996, ApJ, 473, 365

Kaastra, J. S., \& Mewe, R. 2000, in Atomic Data Needs for X-ray Astronomy, p. 161

Klein, R. I., McKee, C. F., \& Colella, P. 1994, ApJ, 420, 213

Lazarian, A. 2006, ApJ, 645, L25

Löhner, R. 1987, Comp. Meth. Appl. Mech. Eng., 61, 323

Mac Low, M., McKee, C. F., Klein, R. I., Stone, J. M., \& Norman, M. L. 1994, ApJ, 433, 757

Mac Low, M.-M., \& Klessen, R. S. 2004, Reviews of Modern Physics, 76, 125
MacNeice, P., Olson, K. M., Mobarry, C., de Fainchtein, R., \& Packer, C. 2000, Comp. Phys. Comm., 126, 330

Mellema, G., Kurk, J. D., \& Röttgering, H. J. A. 2002, A\&A, 395, L13

Mewe, R., Gronenschild, E. H. B. M., \& van den Oord, G. H. J. 1985, A\&AS, 62, 197

Miceli, M., Bocchino, F., Maggio, A., \& Reale, F. 2005, A\&A, 442, 513

Miceli, M., Reale, F., Orlando, S., \& Bocchino, F. 2006, A\&A, 458, 213

Nakamura, F., McKee, C. F., Klein, R. I., \& Fisher, R. T. 2006, ApJS, 164, 477

Narayan, R., \& Medvedev, M. V. 2001, ApJ, 562, L129

Orlando, S. Bocchino, F., Peres, G., Reale, F., Plewa, T., \& Rosner, R. 2006, A\&A, 457, 545

Orlando, S., Peres, G., Reale, F., Bocchino, F., Rosner, R., Plewa, T., \& Siegel, A. 2005, A\&A, 444, 505

Pagano, P., Reale, F., Orlando, S., \& Peres, G. 2007, A\&A, 464, 753

Patnaude, D. J., Fesen, R. A., Raymond, J. C., Levenson, N. A., Graham, J. R., \& Wallace, D. J. 2002, AJ, 124, 2118

Raymond, J. C., \& Smith, B. W. 1977, ApJS, 35, 419

Spitzer, L. 1962, Physics of Fully Ionized Gases (New York: Interscience, 1962)

Zel'dovich, Y. B., \& Raizer, Y. P. 1966, Physics of Shock Waves and High-Temperature Hydrodynamic Phenomena (New York: Academic Press, 1966) 